\title{
Spectroscopic and crystallographic study of 27,28-diethoxy-p-tert-butylcalix[4]arenes
}

\author{
J.A. Kanters ${ }^{\mathrm{a}}$, A. Schouten ${ }^{\mathrm{a}}$, E. Steinwender ${ }^{\mathrm{b}}$, J.H. van der Maas ${ }^{\mathrm{b}}$, \\ L.C. Groenen ${ }^{c}$ and D.N. Reinhoudt ${ }^{c}$ \\ ${ }^{\mathrm{D}}$ Department of Crystal and Structural Chemistry, Bijvoet Center for Biomolecular \\ Research, University of Utrecht, Transitorium 3, Padualaan 8, 3584 CH Utrecht \\ (The Netherlands) \\ ${ }^{\mathrm{b}}$ Department of Analytical Molecular Spectrometry, Faculty of Chemistry, University of \\ Utrecht, P.O. Box 80083, 3508 TB Utrecht (The Netherlands) \\ 'Laboratory of Organic Chemistry, University of Twente, P.O. Box 217, 7500 AE Enschede \\ (The Netherlands)
}

(Received 20 November 1991)

\begin{abstract}
$\mathrm{C}_{48} \mathrm{H}_{64} \mathrm{O}_{4}, M_{\mathrm{r}}=705.04$, monoclinic, $P 2_{1} / n, a=14.509(2) \AA, b=17.913(4) \AA, c=16.899(1) \AA$, $\beta=107.343(9)^{\circ}, V=4192.4(11) \AA^{3}, Z=4, D_{\mathrm{x}}=1.117 \mathrm{~g} \mathrm{~cm}^{-3}, \lambda($ Mo K $\alpha)=0.71073 \AA, \mu=$ $0.74 \mathrm{~cm}^{-1}, F(000)=1536, T=100 \mathrm{~K}, R=0.059$ for 5505 observed reflections with $I>2.5 \sigma(I)$.

The molecular conformation of the anti form of the title compound, i.e. with the two neighbouring phenylethylether fragments in anti positions, is a partial cone. The conformation is stabilized by two intramolecular hydrogen bonds. These bonds involve both the phenolic $\mathrm{OH}$ groups as donors and a hydroxyl group and an ethoxygroup as acceptors. FT-IR solid state spectra reveal two $\mathrm{OH}$ bands at 3388 and $3175 \mathrm{~cm}^{-1}$ which is in accordance with the observed hydrogen-bond configuration. In $\mathrm{CCl}_{4}$ solution the partial cone is preserved for the anti form ( 3378 and $3187 \mathrm{~cm}^{-1}$ ) while the corresponding syn form mainly adopts a cone conformation characterized by $\mathrm{O}-\mathrm{H}$ stretching vibrations at 3360 and $3168 \mathrm{~cm}^{-1}$.
\end{abstract}

\section{INTRODUCTION}

The phenol-formaldehyde cyclic oligomers, called calixarenes, form a class of phenolic macrocycles having methylene bridges at positions ortho to the phenolic $\mathrm{OH}$ groups. The X-ray structures of calixarenes having four [1], five [2], six [3], seven [4], or eight [5] phenolic units have been reported. Of particular interest are the calix[4]arenes since, depending on the degree

Correspondence to: Dr. J.A. Kanters, Department of Crystal and Structural Chemistry, Bijvoet Center for Biomolecular Research, University of Utrecht, Transitorium 3, Padualaan 8, $358 \mathrm{CH}$ Utrecht, The Netherlands.

0022-2860/92/\$05.00 (C) 1992 Elsevier Science Publishers B.V. All rights reserved. 
of substitution of the $\mathrm{OH}$ groups (lower rim) and the presence of bulky para substituents (upper rim), like p-tert-butyl, the conformational flexibility of the macrocycles can be drastically influenced. Although O-unsubstituted calix[4]arenes are conformationally mobile in solution, their solid state structure is characterized by a four-membered ring of strong $\mathrm{O}-\mathrm{H} \cdots \mathrm{O}$ hydrogen bonds which force the calix[4]arene into the so-called cone conformation. Substitution at the lower rim of the calix[4]arenes disrupts the circular hydrogen-bond system, allowing three additional conformations [6] (see Fig. 1). The first is the partial cone which can be derived from the cone by rotation of one phenol residue through the annulus of the molecule. So far only four X-ray structures of calix[4]arenes in the partial cone conformation have been reported [7-10]. The second type, the 1,2-alternate, arises from the cone by rotating two neighbouring phenol fragments. So far only three X-ray structures of this type are known [10-12]. The last is the 1,3-alternate, which originates from rotation of two opposite phenol rings. This type is also rare; only three X-ray structures [11-13] show this conformation.

Recently the crystal structures of two calix[4]arene alkylethers have been reported. One involves a mixed ether, $s y n$-1,2-diethoxy-3,4-dimethoxy-ptert-butylcalix[4]arene ${ }^{a}$ which occurs in the partial cone conformation [10]. Interestingly, two conformations of the second one, p-tert-butylcalix[4]arene tetraethylether, have been reported: a partial cone conformation [14] and the rare 1,2-alternate conformation [10].

In an earlier study the conformations of partially methylated p-tertbutylcalix[4]arene have been investigated by molecular mechanics, X-ray crystallography and ${ }^{1} \mathrm{H}$ NMR spectroscopy [7]. It was found that the mono-, di- and trimethylethers all exist preferentially in a cone conformation. However, no experimental data were reported for the 1,2-dimethylated calix[4]arene.

For the anti-1,2-diethylether, three conformations, partial cone, 1,2alternate and 1,3-alternate, are formally possible. The phenolic $\mathrm{OH}$ groups will tend to establish intramolecular hydrogen bonds, which can only be realized in the partial cone and 1,2-alternate forms. These forms exhibit different hydrogen-bonding schemes: $\mathrm{O}-\mathrm{H} \cdots \mathrm{O}-\mathrm{H} \cdots \mathrm{O}-\mathrm{Et}$ in the partial cone and two $\mathrm{O}-\mathrm{H} \cdots \mathrm{O}-\mathrm{Et}$ bonds in the 1,2-alternate form. Also the syn-1,2diethylether can adopt three different conformations, namely the cone, the partial cone and the 1,2-alternate. Only in the cone can two hydrogen bonds be formed.

\footnotetext{
"In this paper we follow the proposal of Groenen et al. [10] for the nomenclature of substituted calix[4]arenes. The prefix syn indicates that two 0 -substituted substituents are on the same face of the molecule, whereas the prefix anti denotes that two 0 -substituted substituents are on different faces of the molecule.
} 


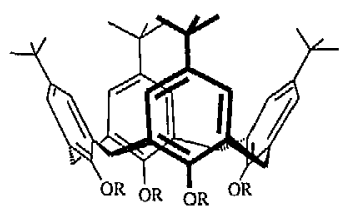

Cone

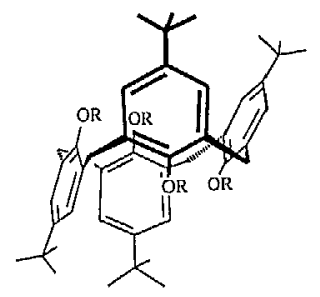

1,2-Alternate

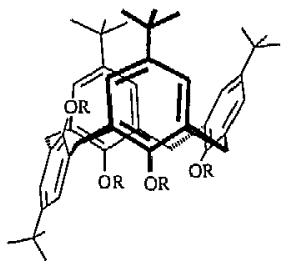

Partial Cone

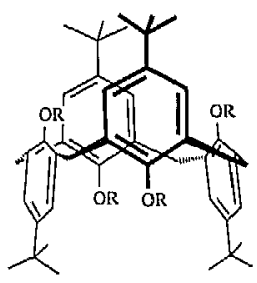

1,3-Alternate

Fig. 1. Schematic drawing of the conformations of calix[4]arenes.

In order to study the balance of conformation-determining effects of these different hydrogen-bonding schemes in the solid state and in solution, we undertook the X-ray analysis and infrared spectroscopy of anti-1,2diethoxy-p-tert-butylcalix[4]arene (ADET). In addition, the solution spectra of the title compound are compared with those of the corresponding syn-1,2diethoxy-p-tert-butylcalix[4]arene (SDET).

\section{EXPERIMENTAL}

\section{Materials}

Crystals of ADET [10] suitable for X-ray analysis were grown from hexane. Crystals of SDET [15] of sufficient quality for X-ray analysis could not be obtained. All solvents used for the solution IR-measurements were of spectroscopic grade and were stored over molecular sieves.

\section{$X$-ray measurements}

A colourless crystal of ADET with dimensions $0.8 \times 0.4 \times 0.2 \mathrm{~mm}^{3}$ was used for data collection on an Enraf-Nonius CAD-4 diffractometer with Zr-filtered Mo $\mathrm{K} \alpha$ radiation. Lattice parameters were determined from the setting angles of 25 reflections in the range $9.24^{\circ} \leqslant \Theta \leqslant 19.23^{\circ}$. The diffracted intensities of 9526 unique reflections were collected at liquid-nitrogen temperature $(100 \mathrm{~K})$ using the $\omega-2 \Theta$ scan mode, $\Delta \omega=(0.67+0.35 \tan \Theta)^{\circ}$, 
$2 \Theta_{\max }=55^{\circ}$ and $-18 \leqslant h \leqslant 18,0 \leqslant k \leqslant 23,-21 \leqslant l \leqslant 0$, of which 5505 with $I>2.5 \sigma(I)$ were considered observed. Three periodically measured standard reflections $(32 \overline{5}, 412$ and $\overline{2} 1 \overline{3})$, measured every hour, showed an average deviation of less than $6 \%$ during 155 hours of X-ray exposure. Intensities were corrected for Lp effects, but not for absorption. The structure was solved in space group $P 2_{1} / n$ by direct methods using sHeLXs86 [16]. The hydroxyl $\mathrm{H}$ atoms were located from a different Fourier map and positionally refined. All other $\mathrm{H}$ atoms were introduced at calculated positions (C-H $1.00 \AA$ ) and refined with respect to their bonded atom, with an overall isotropic thermal parameter which refined to $0.0306(12) \AA^{2}$. Anisotropic full-matrix least-squares refinement on $F$ of 476 parameters converged at $R=0.059$ and $w R=0.062$ with $w=1.687 / \sigma^{2}\left(F_{0}\right), S=1.73$, $(\Delta / \sigma)_{\mathrm{av}}=0.006$ and $(\Delta / \sigma)_{\max }=0.04$. Maximum and minimum residual densities in the final difference map are 0.43 and $-0.36 \mathrm{e}^{-3}$, respectively. The scattering factors were those of Cromer and Mann [17] and anomalousdispersion terms from Cromer and Liberman [18]. The least-squares refinement was performed with the SHELX76 program [19] and the program package EUCLID [20] was used for the calculation of geometries and preparation of illustrations. All calculations were done on an ULTRIX DECsystem-5000.

\section{FT-IR measurements}

The FT-IR spectra were recorded on a Mattson 5020 connected to a PC at a resolution of $2 \mathrm{~cm}^{-1}$. Solution spectra with a concentration less than $2 \mathrm{mg}$ per $5 \mathrm{ml}$ solvent were measured using $10 \mathrm{~mm}$ 'infrasil' cells. For the solid state spectra we used an RIIC-VLT-2 variable temperature unit equipped with $\mathrm{KBr}$ windows connected to a West M2071 microprocessor-based controller. Liquid nitrogen was used as coolant enabling us to obtain fluorolube mull spectra over a temperature range of $100-300 \mathrm{~K}$. Second order derivative spectra were calculated on a PC using the standard Mattson First ${ }^{\mathrm{TM}}$ software.

\section{RESULTS AND DISCUSSION}

\section{Crystal structure}

The atomic coordinates together with equivalent isotropic thermal parameters ( $\mathrm{C}$ and $\mathrm{O}$ atoms) are listed in Table 1. Bond distances and bond angles involving $\mathrm{C}$ and $\mathrm{O}$ atoms are given in Table $2^{\mathrm{b}}$. A perspective view

\footnotetext{
${ }^{\mathrm{b}}$ Non-hydrogen atom anisotropic thermal parameters, coordinates of the hydrogen atoms, and listings of observed and calculated structure factors are available as supplementary data (B.L.L.D. Supplementary Publication number SUP 26443 (61 pages)).
} 


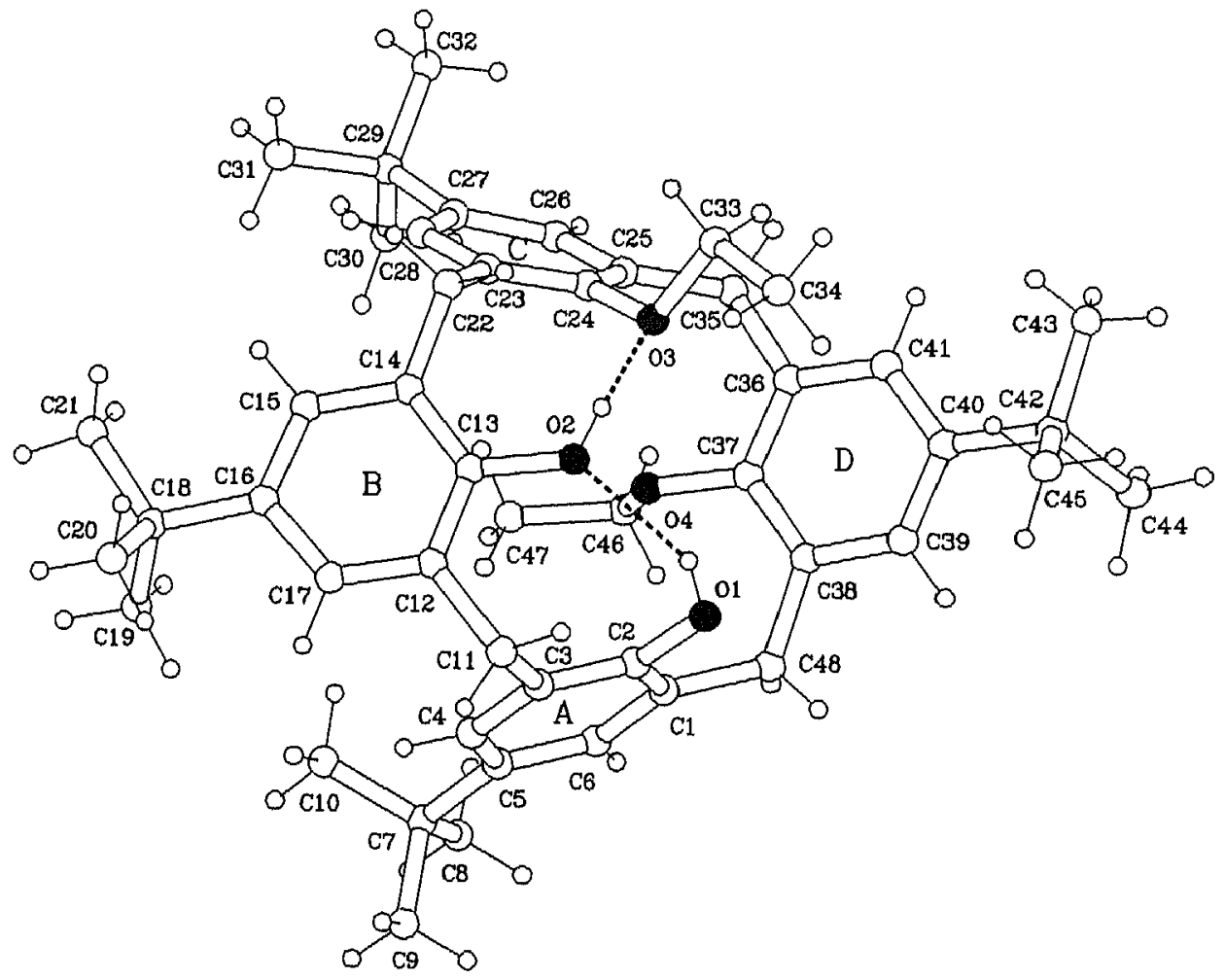

Fig. 2. Perspective view and atomic numbering of the title compound.

of the molecule with the adopted atom numbering scheme is shown in Fig. 2. As Fig. 2 shows, the phenyl ring $D$ is rotated through the calixarene annulus which results in a partial cone conformation. Following the proposal of Groenen et al. [10], the title compound should be classified as an anti-diethoxy-calix [4]arene. In accordance with the partial cone conformation, the distances of the four $\mathrm{O}$ atoms to the plane of the four bridging methylene $C$ atoms are: $1.299(9), 1.087(8), 1.170(8)$ and $-1.101(8) \AA$, respectively. An elegant way to describe the exact conformation of calixarenes was recently proposed by Andreetti et al. [4]. This method circumvents the ambiguities inherent in the common use of the angles between the phenyl rings and the plane of the methylene carbon atoms. Instead the conformation is described by the torsion angles about the $\mathrm{C}-\mathrm{C}$ bonds linking the phenyl rings. For a cone, the sequence of the eight torsion angles have alternate + and - signs. For a partial cone, i.e. when one of the phenyl rings is rotated through the annulus, the torsion angles should display the pattern,,,+-+-++-- or the opposite. As shown in Table 3, the angles about the $\mathrm{C}\left(\mathrm{sp}^{3}\right)-\mathrm{C}\left(\mathrm{sp}^{2}\right)$ bonds uniquely define the conformation as 
TABLE 1

Fractional coordinates and equivalent isotropic thermal parameters $\left(\AA^{2}\right)$ with esd values in parentheses

\begin{tabular}{|c|c|c|c|c|}
\hline Atom & $x$ & $y$ & $z$ & $U_{e q}{ }^{a}$ \\
\hline$O(1)$ & $0.84769(15)$ & $0.27098(11)$ & $0.43904(12)$ & $0.0196(6)$ \\
\hline $\mathrm{O}(2)$ & $0.69852(14)$ & $0.20374(11)$ & $0.31190(12)$ & $0.0180(6)$ \\
\hline$O(3)$ & $0.55353(14)$ & $0.27603(11)$ & $0.33842(12)$ & $0.0175(6)$ \\
\hline $\mathrm{O}(4)$ & $0.68676(14)$ & $0.43053(11)$ & $0.34569(11)$ & $0.0183(6)$ \\
\hline $\mathrm{C}(1)$ & $0.89248(19)$ & $0.39072(16)$ & $0.40390(17)$ & $0.0155(8)$ \\
\hline $\mathrm{C}(2)$ & $0.87911(18)$ & $0.31527(15)$ & $0.38575(17)$ & $0.0154(8)$ \\
\hline$C(3)$ & $0.89937(18)$ & $0.28560(16)$ & $0.31655(17)$ & $0.0153(8)$ \\
\hline $\mathrm{C}(4)$ & $0.93272(18)$ & $0.33264(15)$ & $0.26627(17)$ & $0.0153(8)$ \\
\hline$C(5)$ & $0.94854(18)$ & $0.40807(15)$ & $0.28278(17)$ & $0.0146(8)$ \\
\hline$C(6)$ & $0.92822(19)$ & $0.43623(15)$ & $0.35293(17)$ & $0.0155(8)$ \\
\hline $\mathrm{C}(7)$ & $0.9889(2)$ & $0.45632(17)$ & $0.22580(17)$ & $0.0193(8)$ \\
\hline $\mathrm{C}(8)$ & $0.9892(2)$ & $0.53894(17)$ & $0.24677(19)$ & $0.0251(9)$ \\
\hline $\mathrm{C}(9)$ & $1.0925(2)$ & $0.43206(19)$ & $0.2339(2)$ & $0.0309(11)$ \\
\hline $\mathrm{C}(10)$ & $0.9265(3)$ & $0.44636(19)$ & $0.13499(19)$ & $0.0317(10)$ \\
\hline $\mathrm{C}(11)$ & $0.88112(18)$ & $0.20412(15)$ & $0.29195(16)$ & $0.0148(8)$ \\
\hline$C(12)$ & $0.79077(19)$ & $0.19925(15)$ & $0.21869(17)$ & $0.0149(8)$ \\
\hline $\mathrm{C}(13)$ & $0.70080(19)$ & $0.20491(15)$ & $0.23100(17)$ & $0.0151(8)$ \\
\hline $\mathrm{C}(14)$ & $0.61709(19)$ & $0.21090(15)$ & $0.16364(17)$ & $0.0161(8)$ \\
\hline$C(15)$ & $0.6268(2)$ & $0.21064(16)$ & $0.08349(17)$ & $0.0181(8)$ \\
\hline$C(16)$ & $0.71615(19)$ & $0.20426(16)$ & $0.06952(17)$ & $0.0166(8)$ \\
\hline $\mathrm{C}(17)$ & $0.79748(19)$ & $0.19806(15)$ & $0.13846(17)$ & $0.0163(8)$ \\
\hline$C(18)$ & $0.73016(19)$ & $0.20594(17)$ & $-0.01675(17)$ & $0.0190(8)$ \\
\hline$C(19)$ & $0.7967(2)$ & $0,27082(19)$ & $-0.02204(19)$ & $0.0287(10)$ \\
\hline$C(20)$ & $0.7756(2)$ & $0.13251(19)$ & $-0.03273(19)$ & $0.0289(10)$ \\
\hline $\mathrm{C}(21)$ & $0.6346(2)$ & $0.21630(19)$ & $-0.08485(18)$ & $0.0245(9)$ \\
\hline $\mathrm{C}(22)$ & $0.51732(19)$ & $0.21801(16)$ & $0.17478(17)$ & $0.0168(8)$ \\
\hline$C(23)$ & $0.48870(19)$ & $0.29586(16)$ & $0.19219(17)$ & $0.0152(8)$ \\
\hline $\mathrm{C}(24)$ & $0.50624(19)$ & $0.32329(16)$ & $0.27250(17)$ & $0.0165(8)$ \\
\hline$C(25)$ & $0.48177(19)$ & $0.39456(16)$ & $0.28880(17)$ & $0.0169(8)$ \\
\hline$C(26)$ & $0.4334(2)$ & $0.43928(16)$ & $0.22116(17)$ & $0.0183(8)$ \\
\hline $\mathrm{C}(27)$ & $0.4107(2)$ & $0.41326(17)$ & $0.13930(17)$ & $0.0188(8)$ \\
\hline $\mathrm{C}(28)$ & $0.44023(19)$ & $0.34215(16)$ & $0.12596(17)$ & $0.0174(8)$ \\
\hline$C(29)$ & $0.3510(2)$ & $0.46341(17)$ & $0.06875(18)$ & $0.0226(9)$ \\
\hline $\mathrm{C}(30)$ & $0.4015(3)$ & $0.53840(19)$ & $0.0705(2)$ & $0.0356(11)$ \\
\hline $\mathrm{C}(31)$ & $0.3362(3)$ & $0.4269(2)$ & $-0.01668(18)$ & $0.0339(11)$ \\
\hline $\mathrm{C}(32)$ & $0.2509(2)$ & $0.47592(19)$ & $0.07923(19)$ & $0.0283(10)$ \\
\hline$C(33)$ & $0.4909(2)$ & $0.23990(17)$ & $0.37799(19)$ & $0.0227(9)$ \\
\hline $\mathrm{C}(34)$ & $0.5524(3)$ & $0.18883(18)$ & $0.4443(2)$ & $0.0297(10)$ \\
\hline$C(35)$ & $0.5014(2)$ & $0.42730(17)$ & $0.37551(17)$ & $0.0193(8)$ \\
\hline $\mathrm{C}(36)$ & $0.5964(2)$ & $0.40897(15)$ & $0.44061(17)$ & $0.0165(8)$ \\
\hline$C(37)$ & $0.6845(2)$ & $0.41827(15)$ & $0.42582(17)$ & $0.0162(8)$ \\
\hline $\mathrm{C}(38)$ & $0.77164(19)$ & $0.40767(15)$ & $0.48933(17)$ & $0.0156(8)$ \\
\hline$C(39)$ & $0.7658(2)$ & $0.38339(15)$ & $0.56592(17)$ & $0.0161(8)$ \\
\hline $\mathrm{C}(40)$ & $0.6790(2)$ & $0.36978(15)$ & $0.58197(17)$ & $0.0167(8)$ \\
\hline
\end{tabular}


TABLE 1 (continued)

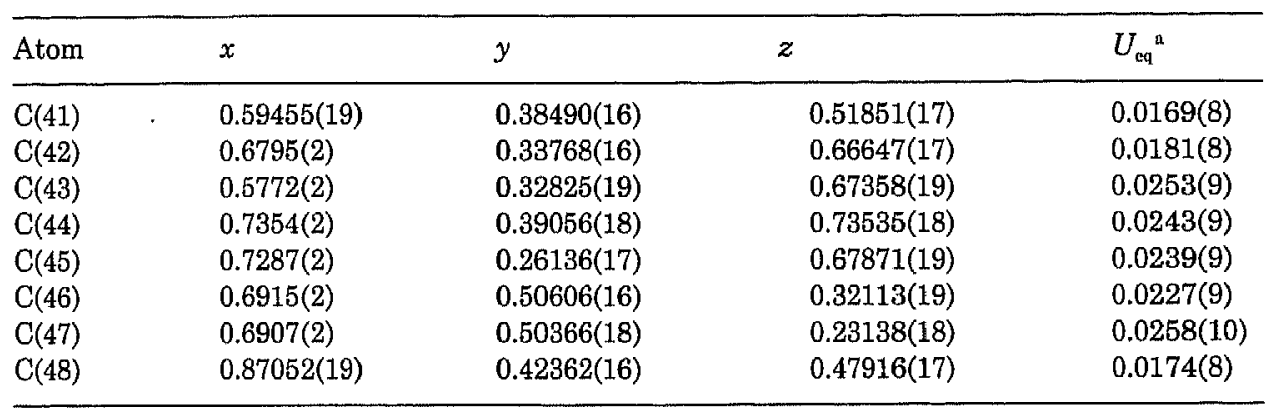

${ }^{\mathrm{a}} U_{\mathrm{eq}}=(1 / 3) \Sigma_{i} \Sigma_{j} U_{i j} a_{i}^{*} a_{j}^{*} a_{i} \cdot \boldsymbol{a}_{j}$.

a partial cone. So it turns out that the crystal-structure conformation of the title compound, a partial cone with two ethoxy groups in the anti position, represents a unique case.

Molecular mechanics calculations for the analogous 1,2-dimethylether also predict that the partial cone is the most stable conformation [7].

The partial cone is strongly deformed as indicated by the interplanar angles of the four phenyl rings with the plane of the methylene $\mathrm{C}$ atoms: for rings $\mathrm{A}, \mathrm{B}, \mathrm{C}$ and $\mathrm{D}$ these angles are 73.6(1), 48.4(1), 58.0(1) and $-67.0(1)^{\circ}$, respectively. Ring $\mathrm{A}$ is nearly perpendicular to rings $\mathrm{B}\left(84.0(1)^{\circ}\right)$ and $\mathrm{D}$ $\left(84.8(1)^{\circ}\right)$, whereas rings $B$ and $D$ approach parallelity $\left(18.7(1)^{\circ}\right)$. The four phenyl rings are planar, the average $\sigma_{\text {plane }}$ is $0.014 \AA$ and the plane of the four linking methylene $\mathrm{C}$ atoms is slightly puckered $\left(\sigma_{\text {plane }}=0.157 \AA\right)$. Three of the tert-butyl groups are staggered with respect to one of the aromatic $\mathrm{C}-\mathrm{C}$ bonds (average torsion angle $176.4^{\circ}$ ) and at ring $\mathrm{B}$ the conformation is eclipsed (torsion angle $\left.0.6(4)^{\circ}\right)$. As in the teraethylether and mixed diethyldimethylethers [10] mentioned earlier, the ethoxy groups are oriented in such a way that the $\mathrm{CH}_{2}$ groups point outwards and the $\mathrm{CH}_{3}$ groups inwards, as follows from the $\mathrm{C}-\mathrm{O}-\mathrm{C}-\mathrm{C}$ torsion angles of $177.4(2)$ and $-178.7(2)^{\circ}$. Three of the bond angles at the bridging methylene carbon atoms are similar $\left(118.8(2), 115.4(2)\right.$ and $\left.117.5(2)^{\circ}\right)$; the angle involving rings $\mathrm{A}$ and $\mathrm{B}$, however, is significantly smaller $\left(108.5(2)^{\circ}\right)$. The partial cone conformation is stabilized by two intramolecular hydrogen bonds. The $\mathrm{O}(1)-\mathrm{H}$ group donates a bond to $\mathrm{O}(2)$ of the second hydroxyl group with geometries $\mathrm{O} \cdots \mathrm{O} 2.825(3), \mathrm{H} \cdots \mathrm{O} 2.09(3) \AA$ and $\mathrm{O}-\mathrm{H} \cdots \mathrm{O}$ 147(3) ${ }^{\circ}$, the $\mathrm{O}(2)-\mathrm{H}$ group donates to $\mathrm{O}(3)$ of a neighbouring ethoxy group with geometries $\mathrm{O} \cdots \mathrm{O} 2.620(3), \mathrm{H} \cdots \mathrm{O}$ 1.71(3) $\AA$ and $\mathrm{O}-\mathrm{H} \cdots \mathrm{O}$ 174(3) 
TABLE 2

Bond distances $(\AA)$ and bond angles (deg.) with esd values in parentheses

\begin{tabular}{|c|c|c|c|}
\hline Parameter & Value & Parameter & Value \\
\hline \multicolumn{4}{|l|}{ Bond length } \\
\hline $\mathrm{O}(1)-\mathrm{C}(2)$ & $1.376(3)$ & $C(18)-C(20)$ & $1.531(4)$ \\
\hline$O(2)-C(13)$ & $1.378(3)$ & $\mathrm{C}(18)-\mathrm{C}(21)$ & $1.526(4)$ \\
\hline $\mathrm{O}(3)-\mathrm{C}(24)$ & $1.405(3)$ & $\mathrm{C}(22)-\mathrm{C}(23)$ & $1.509(4)$ \\
\hline$O(3)-C(33)$ & $1.433(4)$ & $\mathrm{C}(23)-\mathrm{C}(24)$ & $1.394(4)$ \\
\hline $\mathrm{O}(4)-\mathrm{C}(37)$ & $1.382(3)$ & $\mathrm{C}(23)-\mathrm{C}(28)$ & $1.402(4)$ \\
\hline$O(4)-C(46)$ & $1.423(4)$ & $\mathrm{C}(24)-\mathrm{C}(25)$ & $1.375(4)$ \\
\hline$C(1)-C(2)$ & $1.387(4)$ & $\mathrm{C}(25)-\mathrm{C}(26)$ & $1.401(4)$ \\
\hline$C(1)-C(6)$ & $1.393(4)$ & $\mathrm{C}(25)-\mathrm{C}(35)$ & $1.524(4)$ \\
\hline $\mathrm{C}(1)-\mathrm{C}(48)$ & $1.519(4)$ & $\mathrm{C}(26)-\mathrm{C}(27)$ & $1.403(4)$ \\
\hline $\mathrm{C}(2)-\mathrm{C}(3)$ & $1.393(4)$ & $\mathrm{C}(27)-\mathrm{C}(28)$ & $1.384(4)$ \\
\hline$C(3)-C(4)$ & $1.382(4)$ & $\mathrm{C}(27)-\mathrm{C}(29)$ & $1.537(4)$ \\
\hline $\mathrm{C}(3)-\mathrm{C}(11)$ & $1.519(4)$ & $\mathrm{C}(29)-\mathrm{C}(30)$ & $1.526(5)$ \\
\hline $\mathrm{C}(4)-\mathrm{C}(5)$ & $1.385(4)$ & $C(29)-C(31)$ & $1.541(4)$ \\
\hline$C(5)-C(6)$ & $1.398(4)$ & $\mathrm{C}(29)-\mathrm{C}(32)$ & $1.531(4)$ \\
\hline$C(5)-C(7)$ & $1.534(4)$ & $\mathrm{C}(33)-\mathrm{C}(34)$ & $1.514(5)$ \\
\hline$C(7)-C(8)$ & $1.522(4)$ & $\mathrm{C}(35)-\mathrm{C}(36)$ & $1.521(4)$ \\
\hline $\mathrm{C}(7)-\mathrm{C}(9)$ & $1.531(4)$ & $\mathrm{C}(36)-\mathrm{C}(37)$ & $1.384(4)$ \\
\hline$C(7)-C(10)$ & $1.542(4)$ & $\mathrm{C}(36)-\mathrm{C}(41)$ & $1.393(4)$ \\
\hline $\mathrm{C}(11)-\mathrm{C}(12)$ & $1.514(4)$ & $\mathrm{C}(37)-\mathrm{C}(38)$ & $1.406(4)$ \\
\hline$C(12)-C(13)$ & $1.385(4)$ & $\mathrm{C}(38)-\mathrm{C}(39)$ & $1.392(4)$ \\
\hline $\mathrm{C}(12)-\mathrm{C}(17)$ & $1.388(4)$ & $\mathrm{C}(38)-\mathrm{C}(48)$ & $1.522(4)$ \\
\hline$C(13)-C(14)$ & $1,399(4)$ & $\mathrm{C}(39)-\mathrm{C}(40)$ & $1.387(4)$ \\
\hline$C(14)-C(15)$ & $1.403(4)$ & $C(40)-C(41)$ & $1.393(4)$ \\
\hline $\mathrm{C}(14)-\mathrm{C}(22)$ & $1.520(4)$ & $\mathrm{C}(40)-\mathrm{C}(42)$ & $1.537(4)$ \\
\hline$C(15)-C(16)$ & $1.390(4)$ & $\mathrm{C}(42)-\mathrm{C}(43)$ & $1.534(4)$ \\
\hline $\mathrm{C}(16)-\mathrm{C}(17)$ & $1,394(4)$ & $\mathrm{C}(42)-\mathrm{C}(44)$ & $1.532(4)$ \\
\hline $\mathrm{C}(16)-\mathrm{C}(18)$ & $1.531(4)$ & $\mathrm{C}(42)-\mathrm{C}(45)$ & $1.528(4)$ \\
\hline$C(18)-C(19)$ & $1.530(4)$ & $\mathrm{C}(46)-\mathrm{C}(47)$ & $1.514(4)$ \\
\hline \multicolumn{4}{|l|}{ Bond angle } \\
\hline $\mathrm{C}(24)-\mathrm{O}(3)-\mathrm{C}(33)$ & $114.5(2)$ & $\mathrm{C}(13)-\mathrm{C}(14)-\mathrm{C}(22)$ & $122.2(2)$ \\
\hline $\mathrm{C}(37)-\mathrm{O}(4)-\mathrm{C}(46)$ & $117.0(2)$ & $\mathrm{C}(15)-\mathrm{C}(14)-\mathrm{C}(22)$ & $119,6(2)$ \\
\hline $\mathrm{C}(2)-\mathrm{C}(\mathrm{I})-\mathrm{C}(6)$ & $119.1(3)$ & $C(14)-C(15)-C(16)$ & $122.1(3)$ \\
\hline $\mathrm{C}(2)-\mathrm{C}(1)-\mathrm{C}(48)$ & $120.9(3)$ & $\mathrm{C}(15)-\mathrm{C}(16)-\mathrm{C}(17)$ & $117.7(3)$ \\
\hline $\mathrm{C}(6)-\mathrm{C}(1)-\mathrm{C}(48)$ & $120.0(2)$ & $\mathrm{C}(15)-\mathrm{C}(16)-\mathrm{C}(18)$ & $123.7(2)$ \\
\hline $\mathrm{O}(1)-\mathrm{C}(2)-\mathrm{C}(1)$ & $118.0(2)$ & $\mathrm{C}(17)-\mathrm{C}(16)-\mathrm{C}(18)$ & $118.5(3)$ \\
\hline $\mathrm{O}(1)-\mathrm{C}(2)-\mathrm{C}(3)$ & $121.6(2)$ & $\mathrm{C}(12)-\mathrm{C}(17)-\mathrm{C}(16)$ & $121.8(3)$ \\
\hline $\mathrm{C}(1)-\mathrm{C}(2)-\mathrm{C}(3)$ & $120.5(3)$ & $\mathrm{C}(16)-\mathrm{C}(18)-\mathrm{C}(19)$ & $109.6(2)$ \\
\hline $\mathrm{C}(2)-\mathrm{C}(3)-\mathrm{C}(4)$ & $118.9(3)$ & $C(16)-C(18)-C(20)$ & $109.7(2)$ \\
\hline $\mathrm{C}(2)-\mathrm{C}(3)-\mathrm{C}(11)$ & $122.2(2)$ & $\mathrm{C}(16)-\mathrm{C}(18)-\mathrm{C}(21)$ & $111.8(2)$ \\
\hline $\mathrm{C}(4)-\mathrm{C}(3)-\mathrm{C}(11)$ & $118.8(2)$ & $\mathrm{C}(19)-\mathrm{C}(18)-\mathrm{C}(20)$ & $109.3(2)$ \\
\hline $\mathrm{C}(3)-\mathrm{C}(4)-\mathrm{C}(5)$ & $122.6(3)$ & $\mathrm{C}(19)-\mathrm{C}(18)-\mathrm{C}(21)$ & $108.1(2)$ \\
\hline $\mathrm{C}(4)-\mathrm{C}(5)-\mathrm{C}(6)$ & $117.3(2)$ & $C(20)-C(18)-C(21)$ & $108.3(2)$ \\
\hline$C(4)-C(5)-C(7)$ & $119.6(2)$ & $\mathrm{C}(14)-\mathrm{C}(22)-\mathrm{C}(23)$ & $115.4(2)$ \\
\hline
\end{tabular}


TABLE 2 (continued)

\begin{tabular}{llll}
\hline Parameter & Value & Parameter & Value \\
\hline $\mathrm{C}(6)-\mathrm{C}(5)-\mathrm{C}(7)$ & $123.0(2)$ & $\mathrm{C}(22)-\mathrm{C}(23)-\mathrm{C}(24)$ & $122.3(2)$ \\
$\mathrm{C}(1)-\mathrm{C}(6)-\mathrm{C}(5)$ & $121.6(3)$ & $\mathrm{C}(22)-\mathrm{C}(23)-\mathrm{C}(28)$ & $119.5(2)$ \\
$\mathrm{C}(5)-\mathrm{C}(7)-\mathrm{C}(8)$ & $112.1(2)$ & $\mathrm{C}(24)-\mathrm{C}(23)-\mathrm{C}(28)$ & $118.1(3)$ \\
$\mathrm{C}(5)-\mathrm{C}(7)-\mathrm{C}(9)$ & $109.4(2)$ & $\mathrm{O}(3)-\mathrm{C}(24)-\mathrm{C}(23)$ & $117.7(2)$ \\
$\mathrm{C}(5)-\mathrm{C}(7)-\mathrm{C}(10)$ & $109.8(3)$ & $\mathrm{O}(3)-\mathrm{C}(24)-\mathrm{C}(25)$ & $119.7(2)$ \\
$\mathrm{C}(8)-\mathrm{C}(7)-\mathrm{C}(9)$ & $108.7(3)$ & $\mathrm{C}(23)-\mathrm{C}(24)-\mathrm{C}(25)$ & $122.6(3)$ \\
$\mathrm{C}(8)-\mathrm{C}(7)-\mathrm{C}(10)$ & $107.7(2)$ & $\mathrm{C}(24)-\mathrm{C}(25)-\mathrm{C}(26)$ & $117.6(3)$ \\
$\mathrm{C}(9)-\mathrm{C}(7)-\mathrm{C}(10)$ & $109.0(3)$ & $\mathrm{C}(24)-\mathrm{C}(25)-\mathrm{C}(35)$ & $124.4(3)$ \\
$\mathrm{C}(3)-\mathrm{C}(11)-\mathrm{C}(12)$ & $108.5(2)$ & $\mathrm{C}(26)-\mathrm{C}(25)-\mathrm{C}(35)$ & $118.0(3)$ \\
$\mathrm{C}(11)-\mathrm{C}(12)-\mathrm{C}(13)$ & $119.9(2)$ & $\mathrm{C}(25)-\mathrm{C}(26)-\mathrm{C}(27)$ & $121.9(3)$ \\
$\mathrm{C}(11)-\mathrm{C}(12)-\mathrm{C}(17)$ & $120.3(3)$ & $\mathrm{C}(26)-\mathrm{C}(27)-\mathrm{C}(28)$ & $118.3(3)$ \\
$\mathrm{C}(13)-\mathrm{C}(12)-\mathrm{C}(17)$ & $119.4(3)$ & $\mathrm{C}(26)-\mathrm{C}(27)-\mathrm{C}(29)$ & $118.9(3)$ \\
$\mathrm{O}(2)-\mathrm{C}(13)-\mathrm{C}(12)$ & $116.8(2)$ & $\mathrm{C}(28)-\mathrm{C}(27)-\mathrm{C}(29)$ & $122.8(2)$ \\
$\mathrm{O}(2)-\mathrm{C}(13)-\mathrm{C}(14)$ & $122.5(3)$ & $\mathrm{C}(23)-\mathrm{C}(28)-\mathrm{C}(27)$ & $121.4(3)$ \\
$\mathrm{C}(12)-\mathrm{C}(13)-\mathrm{C}(14)$ & $120.8(3)$ & $\mathrm{C}(27)-\mathrm{C}(29)-\mathrm{C}(30)$ & $110.1(3)$ \\
$\mathrm{C}(13)-\mathrm{C}(14)-\mathrm{C}(15)$ & $118.2(3)$ & $\mathrm{C}(27)-\mathrm{C}(29)-\mathrm{C}(31)$ & $111.6(3)$ \\
$\mathrm{C}(27)-\mathrm{C}(29)-\mathrm{C}(32)$ & $109.3(2)$ & $\mathrm{C}(39)-\mathrm{C}(38)-\mathrm{C}(48)$ & $119.0(2)$ \\
$\mathrm{C}(30)-\mathrm{C}(29)-\mathrm{C}(31)$ & $108.9(3)$ & $\mathrm{C}(38)-\mathrm{C}(39)-\mathrm{C}(40)$ & $123.2(3)$ \\
$\mathrm{C}(30)-\mathrm{C}(29)-\mathrm{C}(32)$ & $109.6(3)$ & $\mathrm{C}(39)-\mathrm{C}(40)-\mathrm{C}(41)$ & $117.2(3)$ \\
$\mathrm{C}(31)-\mathrm{C}(29)-\mathrm{C}(32)$ & $107.4(3)$ & $\mathrm{C}(39)-\mathrm{C}(40)-\mathrm{C}(42)$ & $119.6(3)$ \\
$\mathrm{O}(3)-\mathrm{C}(33)-\mathrm{C}(34)$ & $107.6(3)$ & $\mathrm{C}(41)-\mathrm{C}(40)-\mathrm{C}(42)$ & $123.2(3)$ \\
$\mathrm{C}(25)-\mathrm{C}(35)-\mathrm{C}(36)$ & $118.8(2)$ & $\mathrm{C}(36)-\mathrm{C}(41)-\mathrm{C}(40)$ & $121.8(3)$ \\
$\mathrm{C}(35)-\mathrm{C}(36)-\mathrm{C}(37)$ & $122.0(2)$ & $\mathrm{C}(40)-\mathrm{C}(42)-\mathrm{C}(43)$ & $112.2(2)$ \\
$\mathrm{C}(35)-\mathrm{C}(36)-\mathrm{C}(41)$ & $118.8(3)$ & $\mathrm{C}(40)-\mathrm{C}(42)-\mathrm{C}(44)$ & $109.2(2)$ \\
$\mathrm{C}(37)-\mathrm{C}(36)-\mathrm{C}(41)$ & $119.1(3)$ & $\mathrm{C}(40)-\mathrm{C}(42)-\mathrm{C}(45)$ & $109.5(2)$ \\
$\mathrm{O}(4)-\mathrm{C}(37)-\mathrm{C}(36)$ & $119.3(3)$ & $\mathrm{C}(43)-\mathrm{C}(42)-\mathrm{C}(44)$ & $108.3(2)$ \\
$\mathrm{O}(4)-\mathrm{C}(37)-\mathrm{C}(38)$ & $119.4(3)$ & $\mathrm{C}(43)-\mathrm{C}(42)-\mathrm{C}(45)$ & $108.4(2)$ \\
$\mathrm{C}(36)-\mathrm{C}(37)-\mathrm{C}(38)$ & $121.0(3)$ & $\mathrm{C}(44)-\mathrm{C}(42)-\mathrm{C}(45)$ & $109.3(2)$ \\
$\mathrm{C}(37)-\mathrm{C}(38)-\mathrm{C}(39)$ & $117.5(3)$ & $\mathrm{O}(4)-\mathrm{C}(46)-\mathrm{C}(47)$ & $11.5(2)$ \\
$\mathrm{C}(37)-\mathrm{C}(38)-\mathrm{C}(48)$ & $123.5(2)$ & $\mathrm{C}(1)-\mathrm{C}(48)-\mathrm{C}(38)$ & \\
\hline & & & \\
\hline
\end{tabular}

\section{Infrared}

The FT-IR spectra of the anti-1,2-diethoxy-p-tert-butylcalix[4]arene (ADET) in fluorolube mull are presented for two different temperatures (300 and $100 \mathrm{~K}$ ) in Fig. 3 for the $\mathrm{OH}$ stretching region. Figures 4 and 5 show ADET and its syn analogue (SDET) in dilute $\mathrm{CCl}_{4}$ and $\mathrm{CS}_{2}$ solution, respectively.

\section{Solid}

Spectra were recorded from ambient temperature down to $100 \mathrm{~K}$ in five steps, firstly to check for solid-solid phase changes and secondly to get 
TABLE 3

Torsion angles (deg.) about the $\mathrm{C}\left(\mathrm{sp}^{3}\right)-\mathrm{C}\left(\mathrm{sp}^{2}\right)$ bonds $\mathrm{s}^{\mathrm{a}}$

\begin{tabular}{lrlr}
\hline Angle & Value & & \\
\hline $\mathrm{C}(4)-\mathrm{C}(3)-\mathrm{C}(11)-\mathrm{C}(12)$ & $-72.9(3)$ & \} & A-B \\
$\mathrm{C}(3)-\mathrm{C}(11)-\mathrm{C}(12)-\mathrm{C}(17)$ & $94.6(3)$ & \} & \\
$\mathrm{C}(15)-\mathrm{C}(14)-\mathrm{C}(22)-\mathrm{C}(23)$ & $-96.7(3)$ & \\
$\mathrm{C}(14)-\mathrm{C}(22)-\mathrm{C}(23)-\mathrm{C}(28)$ & $90.6(3)$ & \\
$\mathrm{C}(26)-\mathrm{C}(25)-\mathrm{C}(35)-\mathrm{C}(36)$ & $-142.4(3)$ & \\
$\mathrm{C}(25)-\mathrm{C}(36)-\mathrm{C}(36)-\mathrm{C}(41)$ & $-131.3(3)$ & $\mathrm{C}-\mathrm{D}$ \\
$\mathrm{C}(39)-\mathrm{C}(38)-\mathrm{C}(48)-\mathrm{C}(1)$ & $132.1(3)$ & \\
$\mathrm{C}(38)-\mathrm{C}(48)-\mathrm{C}(1)-\mathrm{C}(6)$ & $129.2(3)$ & $\mathrm{D}-\mathrm{A}$ \\
\hline
\end{tabular}

${ }^{a}$ Torsion angles all involve a $\mathrm{C}$ atom at the meta position.

information about the temperature dependence of the $\mathrm{OH}$ stretching frequencies for the intramolecular $\mathrm{O}-\mathrm{H} \cdots \mathrm{O}$ hydrogen bonds. The second point seems to be of particular interest since intramolecular $\mathrm{H} \cdots \mathrm{O}$ distances and corresponding $\mathrm{OH}$ frequencies should hardly be influenced by temperature [21]. The spectra in Fig. 3 exhibit two intense bands at 3388.0 and $3175.0 \mathrm{~cm}^{-1}$ at $300 \mathrm{~K}$ and 3394.5 and $3129.0 \mathrm{~cm}^{-1}$ at $100 \mathrm{~K}$. The corresponding halfbandwidths are 41 and $80 \mathrm{~cm}^{-1}$ for ambient temperature and 13 and $32 \mathrm{~cm}^{-1}$ for $100 \mathrm{~K}$. Additionally, we observed a shoulder on the high frequency side of the $3175 \mathrm{~cm}^{-1}$ band at $3217 \mathrm{~cm}^{-1}$ (from second derivative). In the low temperature spectrum (Fig. $3 \mathrm{~b}$ ) this band is not overlapped any more by the $\mathrm{OH}$ mode at $3129 \mathrm{~cm}^{-1}$ and it is therefore assigned to an overtone or combination band.

Frequency shifts relative to the corresponding 'free' $\mathrm{OH}(\Delta \bar{v})$ usually serve as a synonym for hydrogen-bond strength, and bond distances less than the sum of the van der Waals contact radii are taken as geometrical criteria for the formation of hydrogen bonds in solids. The existence of a direct relationship between these quantities has been shown by Novak [22] and Mikenda [23]. For the 'free' phenolic OH group of $p$-tert-butylphenol in $\mathrm{CCl}_{4}$, which can serve as a realistic reference, Lutz and van der Maas [24] observed a stretching frequency of $3613 \mathrm{~cm}^{-1}$ with a halfbandwidth of $16 \mathrm{~cm}^{-1}$. It therefore seems to be straightforward to assign the high frequency band at $3388 \mathrm{~cm}^{-1}\left(\Delta \bar{v}=225 \mathrm{~cm}^{-1}\right)$ to the $\mathrm{O}(1)-\mathrm{H} \cdots \mathrm{O}(2)-\mathrm{H}$ interaction $(\mathrm{O} \cdots \mathrm{O} 2.825 \AA)$ and the low frequency mode at $3175 \mathrm{~cm}^{-1}$ $\left(\Delta \bar{v}=438 \mathrm{~cm}^{-1}\right)$ to the shorter $\mathrm{O}(2)-\mathrm{H} \cdots \mathrm{O}(3)-\mathrm{Et}$ hydrogen bond $(\mathrm{O} \cdots \mathrm{O}$ $2.620 \AA$ ). This assignment is further supported by the unfavourable $\mathrm{O}-\mathrm{H} \cdots \mathrm{O}$ angle $\left(147^{\circ}\right)$ of the $\mathrm{O}(1)-\mathrm{H} \cdots \mathrm{O}(2)$ hydrogen bond which strongly deviates from the preferred linear hydrogen-bond geometry [25, 26]. Especially for this bent hydrogen bond, a drastic decrease of the halfbandwidth can be 


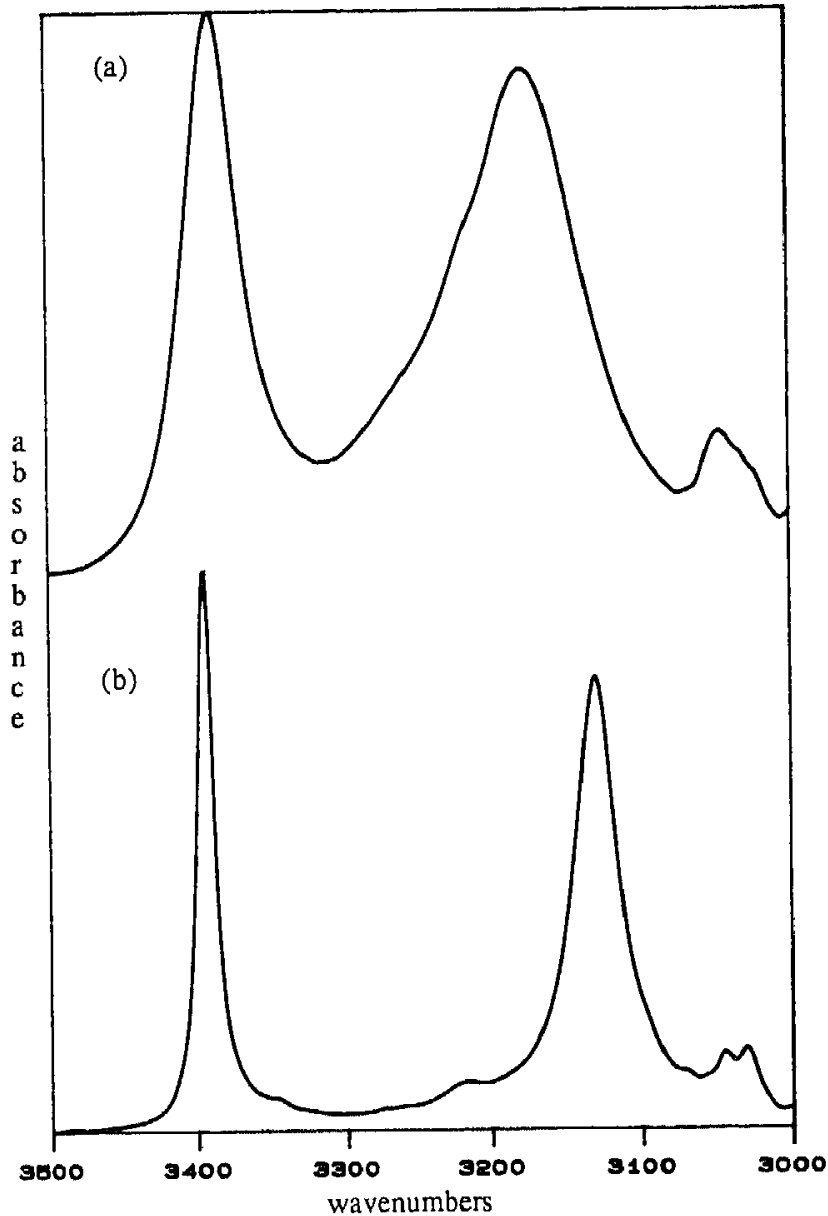

Fig. 3. FT-IR spectra of ADET in fluorolube mull in the $3500-3000 \mathrm{~cm}^{-1}$ range at $300 \mathrm{~K}$ (a) and $100 \mathrm{~K}(\mathrm{~b})$.

observed upon cooling (about 70\%), which is far more than the $40 \%$ found for an intermolecular $\mathrm{O}-\mathrm{H} \cdots \mathrm{O}$ hydrogen bond of similar geometry $(\mathrm{O} \cdots \mathrm{O}$ $2.839 \AA$ and $\left.\mathrm{O}-\mathrm{H} \cdots \mathrm{O} 143^{\circ}\right)$ and frequency $3367 \mathrm{~cm}^{-1}\left(\Delta \bar{v}=246 \mathrm{~cm}^{-1}\right)$ in 2 ethynyladamantan-2-ol [27]. It seems to indicate a better localization of the $\mathrm{O}-\mathrm{H} \cdots \mathrm{O}$ system in the studied compound. Additionally we calculated the temperature coefficients of frequency $\left(\frac{d \bar{v}}{d}\right)$ by plotting the values of the band maxima against temperature and no discontinuity, indicative for a phase change, could be observed. The maximum of the low frequency band shifts from $3175(300 \mathrm{~K})$ to $3129 \mathrm{~cm}^{-1}$ at $100 \mathrm{~K}\left(\frac{\mathrm{d} \overline{\mathrm{r}}}{\mathrm{d} T}=+0.237 \mathrm{~cm}^{-1} \mathrm{~K}^{-1}\right.$; $R^{2}=0.987$ ) whereas that of the high frequency mode shifts slightly in a smooth but non-linear way from $3388(300 \mathrm{~K})$ to $3394.5 \mathrm{~cm}^{-1}(100 \mathrm{~K})$. This 


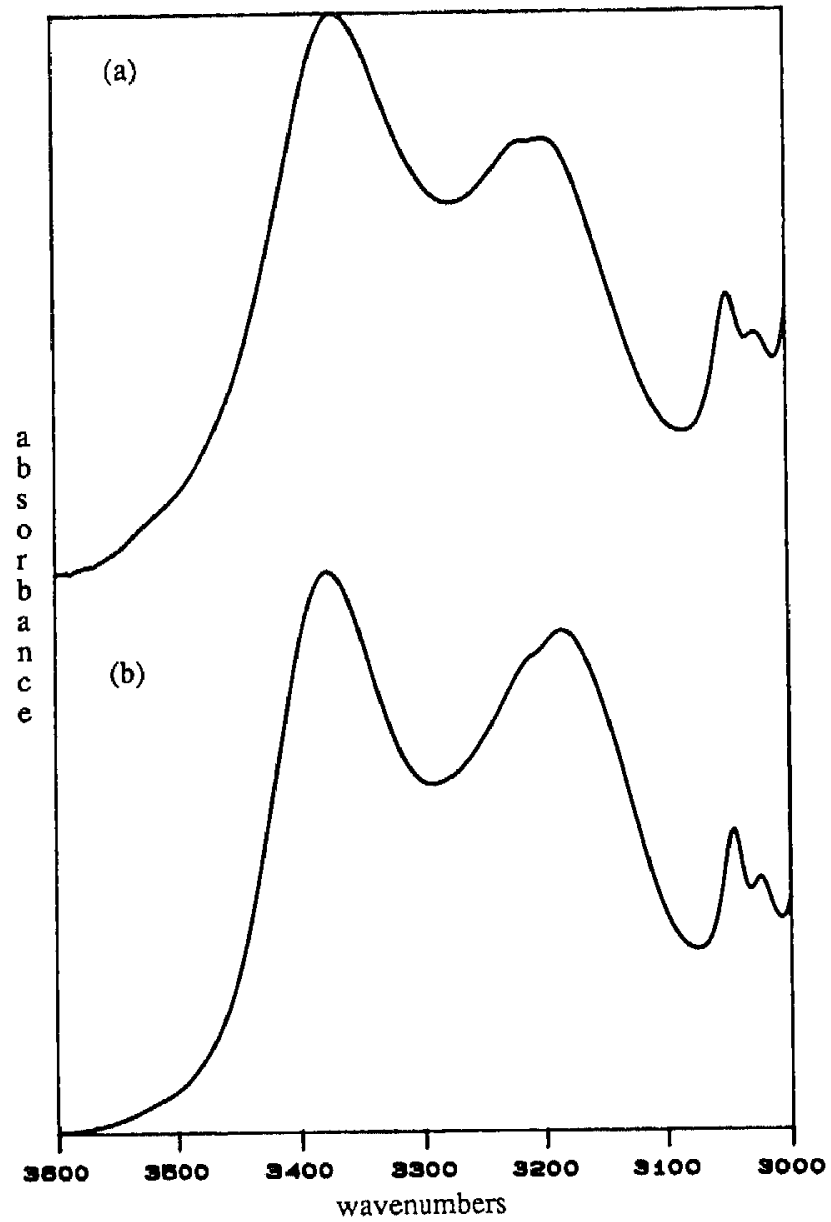

Fig. 4. FT-IR spectra of ADET in the $3600-3000 \mathrm{~cm}^{-1}$ range in $\mathrm{CCl}_{4}$ (a) and $\mathrm{CS}_{2}$ (b).

large positive value is remarkable for an intramolecular hydrogen bond as its order of magnitude is comparable to the values found for intermolecular $\mathrm{O}-\mathrm{H} \cdot \mathrm{O}$ hydrogen-bonded systems of comparable strength $[21,27]$. The main effect causing the low frequency shift of the $\mathrm{OH}$ stretching mode in intermolecular hydrogen bonds upon cooling is thought to be the strengthening of the hydrogen bond by a reduction of the $\mathrm{O} \cdots \mathrm{O}$ distance. Recently Slootmaekers and Desseyn [21] reported that temperature lowering has a much more pronounced effect on the strength of a short hydrogen bond than on a long one. Taking these results into account, together with the opposite sign of the temperature coefficient of frequency for the two intramolecular $\mathrm{O}-\mathrm{H} \cdots \mathrm{O}$ hydrogen bonds in ADET, we conclude that the partial cone, characteristic for the solid state, seems to change its conformation 


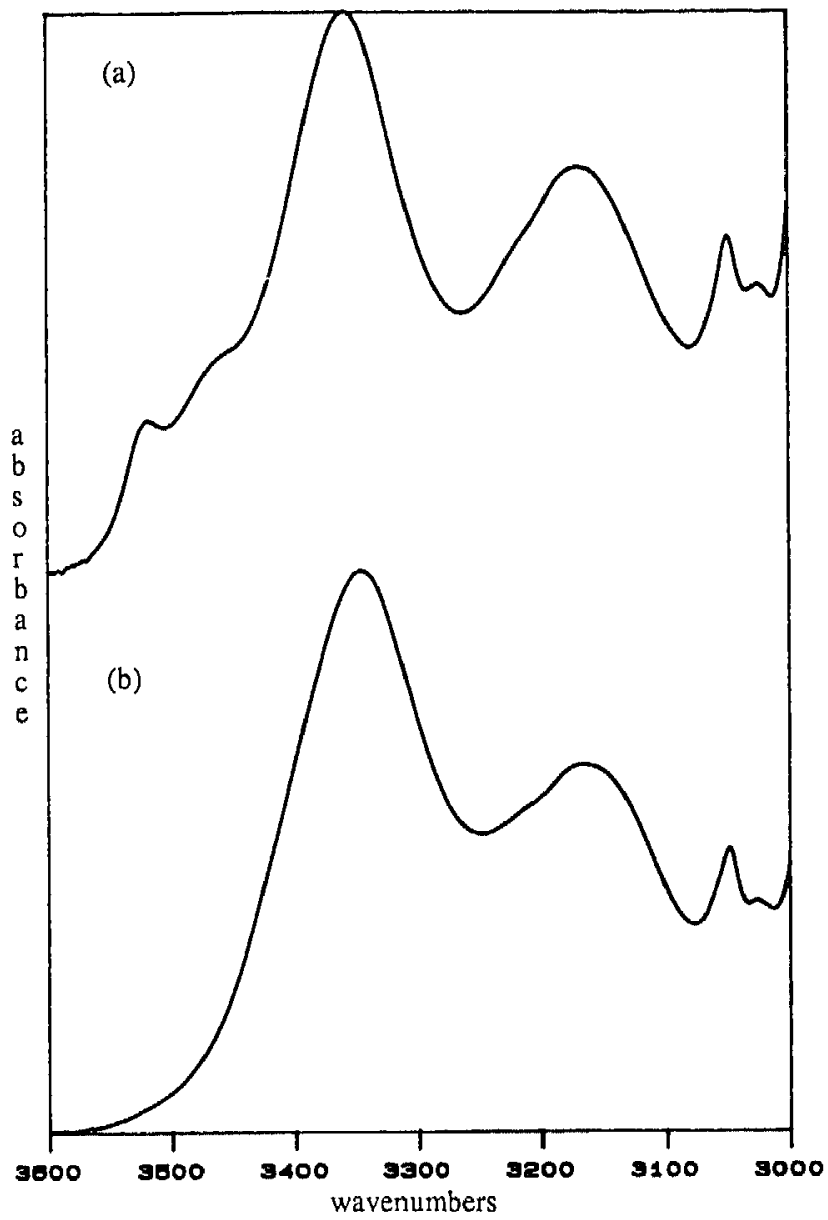

Fig. 5. FT-IR spectra of SDET in the $3600-3000 \mathrm{~cm}^{-1}$ range in $\mathrm{CCl}_{4}$ (a) and $\mathrm{CS}_{2}(\mathrm{~b})$.

slightly upon cooling, with the stronger $\mathrm{O}(2)-\mathrm{H} \cdots \mathrm{O}(3)$ hydrogen bond becoming shorter at the expense of the weaker $\mathrm{O}(1)-\mathrm{H} \cdots \mathrm{O}(2)$ interaction. Another factor worth mentioning might be that the cooperativity in the $\mathrm{O}(1)-\mathrm{H} \cdots \mathrm{O}(2)-\mathrm{H} \cdots \mathrm{O}(3)-\mathrm{Et}$ system becomes more pronounced on cooling because of the reduced thermal vibrations (especially $\mathrm{OH}$ bending modes) and thus favours the $\mathrm{O}(2) \cdots \mathrm{O}(3)-\mathrm{Et}$ hydrogen bond.

\section{Solution}

The solution IR spectra of ADET (Fig. 4) have been measured in $\mathrm{CCl}_{4}$ as well as in $\mathrm{CS}_{2}$ at ambient temperature. Two rather broad, overlapping bands can be observed and therefore we used second derivative spectroscopy for the location of the band maxima. In $\mathrm{CCl}_{4}$ the two $\mathrm{OH}$ stretching 
modes are found at 3378 and $3187 \mathrm{~cm}^{-1}$, and in $\mathrm{CS}_{2}$ at 3386 and $3182 \mathrm{~cm}^{-1}$. Their halfbandwidths range from about 130 to $150 \mathrm{~cm}^{-1}$ in $\mathrm{CCl}_{4}$, but are somewhat smaller in $\mathrm{CS}_{2}$ and much larger than the halfbandwidths of the solid compound. However, the solid state spectra in Fig. 3a, characterizing the partial cone conformation, and the solution spectra (Fig. 4) agree very well in both physical states. This observation leads to the assumption that the partial cone structure, together with a hydrogen-bond geometry comparable to that found for the solid state, is preserved in apolar solution. However, it has been deduced from ${ }^{1} \mathrm{H}$ NMR that ADET is characterized by a 1,2-alternate conformation with one of the ethoxy groups and its neighbouring hydroxyl groups on one side of the ring and the other ethoxy and hydroxyl groups on the other side [10]. This conformer would also exhibit two hydrogen bonds, but the following points support the partial cone structure.

(1) The identity of the band maxima in solid phase and solution.

(2) The $0 \cdots O$ distance of the ether groups on the same and opposite sides of the ring in tetrasubstituted calix[4]arenes adopting the 1,2-alternate conformation in solid state proves to be equal [11] or very similar [10]. Even if the hypothetical 1,2-alternate of ADET would exhibit asymmetry of the two $\mathrm{O}-\mathrm{H} \cdots \mathrm{O}-\mathrm{Et}$ hydrogen bonds, the difference in frequency observed in solution (about $200 \mathrm{~cm}^{-1}$ ) is exceptionally large.

(3) So far $\bar{v}_{\mathrm{OH}}$ values lower than $3200 \mathrm{~cm}^{-1}$ have only been found for calix[4]arenes when at least two neighbouring $\mathrm{OH}$ groups form an $\mathrm{O}-\mathrm{H} \cdots \mathrm{O}-\mathrm{H}$ hydrogen-bond system in which the $\mathrm{O}-\mathrm{H} \cdots \mathrm{O}$ hydrogen bonds are strengthened by cooperativity [28].

(4) For isolated $\mathrm{O}-\mathrm{H} \cdots \mathrm{O}-\mathrm{Me}$ hydrogen bonds in 1,3-dimethoxy-p-tertbutylcalix[4]arene, Araki et al. [28] report an $\mathrm{OH}$ stretching frequency of $3450 \mathrm{~cm}^{-1}$.

An alternative interpretation of the ${ }^{1} \mathrm{H}$ NMR spectrum could be that the observed signals are the time-averaged signals of two equivalent partial cones that are interconverting by simultaneous rotation of both phenol rings.

Considering the above-mentioned results for the anti-1,2-diethylether, one would expect a cone conformation for the corresponding syn-1,2diethylether (SDET) with two different $\mathrm{O}-\mathrm{H} \cdots \mathrm{O}$ hydrogen bonds. Indeed Fig. 5 shows two different $\mathrm{OH}$ stretching bands for SDET in $\mathrm{CCl}_{4}$ (3360 and $3168 \mathrm{~cm}^{-1}$ ) as well as in $\mathrm{CS}_{2}\left(3345\right.$ and $\left.3165 \mathrm{~cm}^{-1}\right)$. The somewhat lower wavenumbers compared to ADET indicate that the cone structure seems to be more symmetric and favours stronger hydrogen bonds than the partial cone structure of the corresponding anti compound. Furthermore, the spectrum of SDET in $\mathrm{CCl}_{4}$ (Fig. 5a) exhibits two additional bands at 3525 and $3468 \mathrm{~cm}^{-1}$ of low intensity, which overlap with the broad absorption on the high frequency side. Surprisingly, both bands are not present in the $\mathrm{CS}_{2}$ 
solution spectrum (Fig. 5b). This brings us to the assumption that SDET adopts, next to the dominating cone, another conformation in $\mathrm{CCl}_{4}$, resulting from the rotation of one or both $\mathrm{OH}$ groups through the annulus of the calix[4]arene. Two distinct conformers could result, a partial cone or a 1,2-alternate, both characterized by a different hydrogen-bonding scheme. The partial cone would exhibit an $\mathrm{O}-\mathrm{H} \cdots \mathrm{O}-\mathrm{Et}$ hydrogen bond and a 'free' $\mathrm{OH}$ group or an $\mathrm{OH}$ group hydrogen-bonded to the $\pi$-system of the neighbouring phenyl rings $(\mathrm{O}-\mathrm{H} \cdots \pi)$, while the 1,2 -alternate would be characterized by one $\mathrm{O}-\mathrm{H} \cdots \mathrm{O}-\mathrm{H}$ hydrogen bond and one 'free' or $\mathrm{O}-\mathrm{H} \cdots \pi$-bonded $\mathrm{OH}$ group. Although the observed frequency of $3525 \mathrm{~cm}^{-1}$ agrees well with the usual values found for $\mathrm{O}-\mathrm{H} \cdots \pi$ hydrogen bonding [29], it is not possible to assign the $3468 \mathrm{~cm}^{-1}$ band unambiguously to an $\mathrm{O}-\mathrm{H} \cdots \mathrm{O}-\mathrm{Et}$ or $\mathrm{O}-\mathrm{H} \cdots \mathrm{O}-\mathrm{H}$ hydrogen bond, thus leaving open the question about the nature of the second conformation of SDET in $\mathrm{CCl}_{4}$ for further discussion.

The striking difference of the $\mathrm{CCl}_{4}$ and $\mathrm{CS}_{2}$ spectra can be understood taking into account that p-tert-butylcalix[4]arenes contain enforced cavities large enough to admit solvent molecules [30]. The limited diameter of these cylindrical wells denies occupancy to the larger $\mathrm{CCl}_{4}$ molecule but allows $\mathrm{CS}_{2}$ to penetrate deeply into the host cavity, as was shown by Cram et al. [31] for similar cavitands and $\mathrm{CS}_{2}$. Cram et al. [32] also stated that most host-guest complexes are more rigid than the host and guests taken separately, which, applied to our SDET- $\mathrm{CS}_{2}$ system, would mean that the cone conformation in solution is not solely stabilized by intramolecular hydrogen bonds but also via the formation of a host-guest complex with the $\mathrm{CS}_{2}$ inside the apolar cavity.

\section{CONCLUSIONS}

In this paper we have tried to clarify how the conformation of $p$-tert-butylcalix[4]arene changes upon partial 1,2-di-O-ethyl substitution. Infrared solution spectra indicate that the 1,2-syn isomer favours the cone conformation, which is stabilized by the formation of two intramolecular hydrogen bonds. Intramolecular hydrogen bonding is also the driving force in establishing the cone conformation of the 1,3-syn isomer [10]. In the 1,2-anti isomer, of which the crystal structure is reported, a cone is impossible because of the anti arrangement of the two ethoxy-phenyl groups. Instead, the compound is seen to exist in the partial cone conformation, which in contrast to the also possible 1,2-alternate form, allows the formation of a cooperative system of two intramolecular hydrogen bonds.

Infrared solution and solid state spectra of the 1,2-anti isomer are in agreement with the observed crystal-structure conformation. The discrepancy between the interpretation of infrared solution spectra and ${ }^{1} \mathrm{H}$ NMR spectra, which indicated a 1,2-alternate [10], may be explained by the 
interconversion of equivalent partial cones, a process which is frozen out on the IR time scale, but not on the ${ }^{1} \mathrm{H}$ NMR time scale.

\section{REFERENCES}

1 G.D. Andreetti, A. Pochini and R. Ungaro, J. Chem. Soc., Perkin Trans., 2 (1984) 1979.

2 M. Corruzzi, G.D. Andreetti, V. Bocchi and R. Ungaro, J. Chem. Soc., Perkin Trans., 2 (1982) 1133.

3 G.D. Andreetti, F. Ugozzoli, A. Casnati, E. Ghidini, A. Pochini and R. Ungaro, Gazz. Chim. Ital., 119 (1989) 47.

4 G.D. Andreetti, F. Ugozzoli, Y. Nakamoto and S.I. Ichida, J. Inclusion Phenom., 10 (1991) 241.

5 C.D. Gutsche, D.A. Gutsche and I. Karaulov, J. Inclusion Phenom., 3 (1985) 447.

6 J.D. van Loon, L.C. Groenen, S.S. Wijmenga, W. Verboom and D.N. Reinhoudt, J. Am. Chem. Soc., 113 (1991) 2378.

7 P.D.J. Grootenhuis, P.A. Kollmann, L.C. Groenen, D.N. Reinhoudt, G.J. van Hummel, F. Ugozzoli and G.D. Andreetti, J. Am. Chem. Soc., 112 (1990) 4165.

8 C. Rizzoli, G.D. Andreetti, R. Ungaro and A. Pochini, J. Mol. Struct., 82 (1982) 133.

9 F. Hamada, S.G. Bott, G.W. Orr, A.W. Coleman, H. Zhang and J.L. Atwood, J. Inclusion Phenom., 9 (1990) 195.

10 L.C. Groenen, J.D. van Loon, W. Verboom, S. Harkema, A. Casnati, R. Ungaro, A. Pochini, F. Ugozzoli and D.N. Reinhoudt, J. Am. Chem. Soc., 113 (1991) 2385.

11 S.G. Bott, A.W. Coleman and J.L. Atwood, J. Inclusion Phenom., 5 (1987) 747.

12 C.D. Gutsche, J.S. Roger and D. Stewart, Pure Appl. Chem., 62 (1990) 485.

13 A. Vrielink, P. Codding, C.D. Gutsche and L.G. Lin, J. Inclusion Phenom., 4 (1986) 199.

14 K. Iwamoto, K. Araki and S. Shinkai, J. Org. Chem., 56 (1991) 4955.

15 L.C. Groenen, B.H.M. Ruël, A. Casnati, P. Timmerman, W. Verboom, S. Harkema, A. Pochini, R. Ungaro and D.N. Reinhoudt, Tetrahedron Lett., 32 (1991) 2675.

16 G.M. Sheldrick, SHELXs86, Program for the solution of crystal structures, University of Göttingen, Germany, 1986.

17 D.T. Cromer and J.B. Mann, Acta Crystallogr., Sect. A, 24 (1968) 321.

18 D.T. Cromer and D. Liberman, J. Chem. Phys., 53 (1970) 1891.

19 G.M. Sheldrick, SHELX76, Program for crystal structure determination, University of Cambridge, England, 1976.

20 A.L. Spek, in D. Sayre (Ed.), The EucLID package in Computational Crystallography, Clarendon Press, Oxford, 1982, p. 528.

21 B. Slootmaekers and H.O. Desseyn, Appl. Spectrosc., 45 (1991) 118.

22 A. Novak, in Structure and Bonding, Vol. 18, Springer-Verlag, Berlin, 1974, p. 177.

23 W. Mikenda, J. Mol. Struct., 147 (1986) 1.

24 E.T.G. Lutz and J.H. van der Maas, Spectrochim. Acta, Part A, 39 (1983) 1007.

25 C. Ceccarelli, G.A. Jeffrey and R. Taylor, J. Mol. Struct., 70 (1981) 255.

26 W.A.P. Luck, in P. Schuster, G. Zundel and C. Sandorfy (Ed.), The Hydrogen Bond, North-Holland, Amsterdam, 1976, p. 527.

27 E. Steinwender, E.T.G. Lutz, J.H. van der Maas and J.A. Kanters, Vib. Spectrosc,, submitted for publication.

28 K. Araki, K. Iwamoto, S. Shinkai and T. Matsuda, Bull. Chem. Soc. Jpn., 63 (1990) 3480.

29 L.J. Bellamy, Advances in Infrared Group Frequencies, Methuen, New York, 1968, p. 246.

30 L.J. Bauer and C.D. Gutsche, J. Am. Chem. Soc., 107 (1985) 6063.

31 D.J. Cram, K.D. Stewart, I. Goldberg and K.N. Trueblood, J. Am. Chem. Soc., 107 (1985) 2574.

32 D.J. Cram, S. Karbach, H.-E. Kim, C.B. Knobler, E.M. Maverick, J.L. Ericson and R.C. Helgeson, J. Am. Chem. Soc., 110 (1988) 2229. 